Polymer Journal, Vol. 3, No. 6, pp 739-748 (1972)

\title{
Randomly Branched Polymers. II. Computer Analysis of Gel-Permeation Chromatogram*
}

\author{
Michio Kurata, ${ }^{* *}$ Hiroyoshi OKamoto, Masamichi Iwama, ${ }^{* * *}$ \\ Mitsuo AвE, ${ }^{* * *}$ and Terutake HommA ${ }^{* * *}$ \\ Institute for Chemical Research, Kyoto University, \\ Uji, Kyoto 611, Japan.
}

(Received February 25, 1972)

\begin{abstract}
An iterative computer method was proposed for estimating the degree of branching and molecular weight distribution simultaneously from a pair of measurements on intrinsic viscosity and gel-permeation chromatography. The validity of the method as applied to randomly branched polymers was tested by using both fractionated and unfractionated samples of branched polystyrenes. It was experimentally concluded that the average number of branch points per unit molecular weight, $\lambda$, can be determined by this method with accuracy of about $15 \%$, and the weight-average molecular weight with accuracy of about $10 \%$.

KEY WORDS Branched Polymer / Polystyrene / Degree of Chain Branching / Computer Analysis / Intrinsic Viscosity / Gel-Permeation Chromatography / Molecular Weight Distribution / Weight-Average Molecular Weight / Kilb Theory /
\end{abstract}

It has been found by Grubisic and others ${ }^{1,2}$ that in the gel-permeation chromatography (hereafter abbreviated as GPC), the effective size of a molecule controlling separation is practically represented by the product of intrinsic viscosity and molecular weight, $Q=[\eta] M$, in either case of linear and branched molecules. This feature has been given a theoretical interpretation, ${ }^{3}$ and now it is widely accepted as the most valuable hypothesis in the study of GPC. The GPC elution curve is thus always convertible to the distribution function of the effective molecular size $Q$ with the aid of a universal calibration curve.

In the case of linear molecules, the distribu-

* This work was supported by the International Institute of Synthetic Rubber Producers, Inc. (IISRP), under contract with the Kyoto University. The main part of this work was reported in "Characterization of Randomly Branched Polymers by Means of Hydrodynamic Measurements and GelPermeation Chromatography," Final Report to IISRP, April 1970.

** To whom correspondence should be addressed.

*** Permanent address: Research Laboratory, Japan Synthetic Rubber Co., Ltd., Mochiizaka, Ikuta, Kawasaki, Japan. tion function of $Q=[\eta] M$ can be separated into two distribution functions of $M$ and [ $\eta]$ by using a viscosity $v s$. molecular weight relationship such that $[\eta]=K M^{A}$. Here $K$ and $A$ are constants. Then, we can evaluate various kinds of average molecular weight, $M_{n}, M_{w}$, and $M_{z}$, and intrinsic viscosity $[\eta]$ of the total sample from these distribution functions of $M$ and [ $\eta$, respectively.

In the case of branched molecules, however, we must know, in advance of the above calculation, the degree of chain branching of the sample, for the $[\eta]$ vs. $M$ relationship to be used depends on this quantity. For a randomly branched polymer with tetra-functional branching units, for example, this relationship is written in the form

$$
[\eta]_{\mathrm{b}}=G_{4}(\lambda, M)[\eta]_{1}=G_{4}(\lambda, M) K M^{A}
$$

with

$$
G_{4}(\lambda, M)=\left[(1+\lambda M / 6)^{1 / 2}+(4 / 3 \pi) \lambda M\right]^{-0.3}
$$

as shown in Part I. Here $\lambda$ is the number of branch points per unit molecular weight of the polymer. Thus, in the treatment of a polymer with unknown degree of branching, we may first assume a trial value of $\lambda$ to fix eq 1 and 
2. Then, using these equations, we derive the distribution function of $[\eta]$ from that of $Q$ and calculate $[\eta]$ of the polymer. In general, such a result may not agree with the observed value of $[\eta]$. Then, the value of $\lambda$ which was used is modified and $[\eta]$ is recalculated. These processes of calculation can be iterated in a computer until agreement is achieved between the calculated and observed values of $[\eta]$. Finally, we can assess simultaneously the degree of branching $\lambda$ and various kinds of average molecular weight.

In this paper, we report the results of the application of the above method to the branched polystyrenes which were prepared in Part I as model samples of randomly branched polymers. After this study was started, we became aware of a report by Drott and Mendelson. ${ }^{4}$ They proposed a method for estimating long-chain branching which was in principle the same as ours, and applied it to low-density polyethylenes. However, no test of the method was made with a laboratory sample bearing a known degree of branching. We believe that such a test is indispensable for elucidating the practical value of the method and for furnishing a firm basis for it.

\section{EXPERIMENTAL}

\section{Polymer Samples}

The samples of branched polymer used were randomly branched polystyrenes prepared by copolymerization of styrene (abbreviated as ST) and 1,4-divinyl-2,3,5,6-tetrachlorobenzene (ab- breviated as DVTCB). In addition to the samples of B-series which were used in Part I, nine other samples, $\mathrm{C}$ to $\mathrm{K}$, were prepared under various conditions of polymerization. Table I summarizes these conditions, i.e., the composition of polymerization mixture, polymerization time, and conversion $\theta$, where the abbreviation AIBN represents $\alpha, \alpha^{\prime}$-azobisisobutyronitrile as an initiator. Polymerization was carried out in benzene at $60^{\circ} \mathrm{C}$. The chlorine content $W_{\mathrm{Cl}}$ and branching parameter $\lambda$ can be evaluated from these data by using eq 3 to 8 in Part I. The results are also shown in Table I. The values of $W_{\mathrm{C} 1}$ are in agreement with the results of chemical analysis within experimental errors.

The new samples, $\mathrm{C}$ to $\mathrm{K}$, were dissolved in benzene, filtered through a GPC filter and then freeze-dried from the solutions. A microgel portion of each original sample was removed by these treatments. Viscosity and GPC measurements were carried out on the filtered samples.

Three kinds of linear polystyrene were also used in this study. The first is the anionically polymerized samples designated as L-series which have a narrow distribution of molecular weight. The properties of these samples have been given in Table III of Part I. The second is a product of bulk polymerization MS-1 which has been distributed from the Committee on Molecular Weight and Molecular Weight Distribution of the Society of Polymer Science, Japan. ${ }^{5}$ The weight-average molecular weight $\boldsymbol{M}_{w}$ has been determined as $(2.3 \pm 0.3) \times 10^{5}$ by several inves-

Table I. Polymerization conditions for branched polystyrene samples

\begin{tabular}{|c|c|c|c|c|c|c|c|c|c|}
\hline \multirow{2}{*}{ Sample code } & \multicolumn{3}{|c|}{ Molar ratio } & \multirow{2}{*}{$\begin{array}{l}\text { Poly- } \\
\text { merization } \\
\text { time, hr }\end{array}$} & \multirow{2}{*}{$\theta$} & \multicolumn{2}{|c|}{$W_{\mathrm{Cl}}$, wt $\%$} & \multirow{2}{*}{$\begin{array}{c}\lambda \\
\times 10^{5}\end{array}$} & \multirow{2}{*}{$\begin{array}{l}{[\eta],} \\
\mathrm{d} l / g\end{array}$} \\
\hline & ST & DVTCB & AIBN & & & Anal. & Calcd & & \\
\hline B & 126 & 1 & 0.0781 & 46 & 0.384 & 1.1 & 1.1 & 1.00 & - \\
\hline $\mathrm{C}$ & 126 & 1 & 0.133 & 66 & 0.446 & 0.9 & 1.1 & 1.23 & 0.639 \\
\hline D & 126 & 1 & 0.0798 & 66 & 0.378 & 1.1 & 1.1 & 0.98 & 0.797 \\
\hline E & 126 & 1 & 0.0221 & 72 & 0.233 & 0.9 & 1.1 & 0.54 & 1.340 \\
\hline F & 126 & 1 & 0.00442 & 72 & 0.140 & 0.8 & 1.1 & 0.30 & 2.220 \\
\hline G & 250 & 1 & 0.0775 & 48.5 & 0.255 & 0.7 & 0.6 & 0.29 & 0.858 \\
\hline $\mathrm{H}$ & 180 & 1 & 0.0558 & 47.5 & 0.261 & 0.6 & 0.8 & 0.42 & 0.935 \\
\hline I & 100 & 1 & 0.0310 & 46 & 0.253 & 1.5 & 1.4 & 0.74 & 1.125 \\
\hline $\mathbf{J}$ & 80 & 1 & 0.0248 & 45 & 0.250 & 1.6 & 1.7 & 0.91 & 1.315 \\
\hline $\mathbf{K}$ & 60 & 1 & 0.0186 & 43 & 0.255 & 2.5 & 2.3 & 1.22 & 1.580 \\
\hline
\end{tabular}


GPC-Computer Analysis of Branched Polymers

Table II. GPC column systems and operating conditions

\begin{tabular}{clccc}
\hline System & Nominal capacity of columns & Solvent & $\begin{array}{c}\text { Temp, } \\
{ }^{\circ} \mathrm{C}\end{array}$ & $\begin{array}{c}\text { Sample concn, } \\
\mathrm{g} / 100 \mathrm{~m} l\end{array}$ \\
\hline I & $10^{4}, 5 \times 10^{5}, 3 \times 10^{6}, 7 \times 10^{6}$ & THF $^{\mathrm{b}}$ & 35 & 0.20 \\
II & $10^{5}, 10^{6}, 10^{7}$, greater than $5 \times 10^{6}$ & THF & 35 & $0.10,0.15$ or 0.20 \\
\hline
\end{tabular}

a Injection time, $90 \mathrm{sec}$; elution rate, $1 \mathrm{ml} / \mathrm{min}$.

b THF, tetrahydrofuran.

Table III. GPC peak count of linear and branched polystyrenes, L- and B-series

\begin{tabular}{cccccl}
\hline $\begin{array}{c}\text { Sample } \\
\text { code }\end{array}$ & $\begin{array}{c}M_{w} \\
\times 10^{-4}\end{array}$ & \multicolumn{2}{c}{$V_{p}$, count } & \multicolumn{1}{c}{$\begin{array}{l}{[\eta],} \\
\mathrm{d} l / \mathrm{g}\end{array}$} & $\begin{array}{c}Q_{w} \\
\times 10^{-6}\end{array}$ \\
\cline { 3 - 5 } L- 7 & 5.1 & 33.11 & 35.54 & 0.295 & 0.0148 \\
L- 1 & 16.5 & 30.24 & 33.63 & 0.668 & 0.1102 \\
L- 3 & 44.4 & 27.70 & 31.57 & 1.32 & 0.586 \\
L- 6 & 87.6 & 25.55 & 29.56 & 2.13 & 1.87 \\
L-14 & 189 & 23.35 & 27.00 & 3.62 & 6.84 \\
B- 2 & 8.96 & 32.20 & 35.00 & 0.360 & 0.0322 \\
B- 3 & 11.8 & 31.20 & 34.31 & 0.461 & 0.0544 \\
B- 4 & 18.9 & 30.37 & 33.67 & 0.627 & 0.1185 \\
B- 5 & 27.2 & 29.50 & 33.00 & 0.776 & 0.211 \\
B- 6 & 39.9 & 28.33 & 32.16 & 0.948 & 0.378 \\
B- 7 & 64.2 & 27.30 & 31.42 & 1.15 & 0.738 \\
B- 8 & 125 & 26.00 & 30.22 & 1.50 & 1.87 \\
B- 9 & 307 & 24.71 & 28.60 & 2.16 & 6.63 \\
\hline
\end{tabular}

tigators, ${ }^{5,6}$ and the ratio of the weight-average to number-average molecular weight, $M_{w} / M_{n}$, has been estimated as $2.3 \pm 0.3$ from sedimentation velocity analysis. ${ }^{6}$ Three samples of the last kind were prepared by radical polymerization in benzene at $60^{\circ} \mathrm{C}$, using AIBN as an initiator, and designated as YML-1, 4, and 7. Their intrinsic viscosities were $0.767,0.443$, and $0.990 \mathrm{~d} l / g$, respectively, as determined in toluene at $30^{\circ} \mathrm{C}$, but no measurement was made for molecular weight.

\section{GPC Measurement}

GPC measurements were carried out in a standard Waters Associates Model 200 gel-permeation chromatograph equipped with a fourcolumn system, I or II. The column capacity and operating conditions are summarized in Table II. Column combination I was a regular one, and was used mainly for analysis of the fractionated samples, B-series. System II was designed to improve resolution in the range of high molecular weight and used for analysis of the unfractionated samples, $\mathrm{C}$ to $\mathrm{K}$.

\section{GPC Calibration Curve}

The elution peak counts $V_{p}$ obtained for the samples of L- and B-series are shown in Table III. There are also shown the weight-average

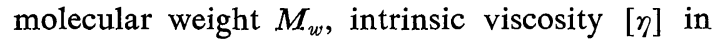
toluene at $30^{\circ} \mathrm{C}$, and their product, $Q_{w}=[\eta] M_{w}$.

Figure 1 shows the plot of $Q_{w}$ against $V_{p}$, where the closed circles represent the data for the linear samples, L-series, and the open circles those for the branched samples, B-series. Except the data points of two samples B-8 and 9 having rather broad distributions of molecular weight, all the points fall on a common calibration curve in each column system, irrespective of chain branching. In system $I$, the calibration curve is linear over the entire range of $Q$ studied and we may put,

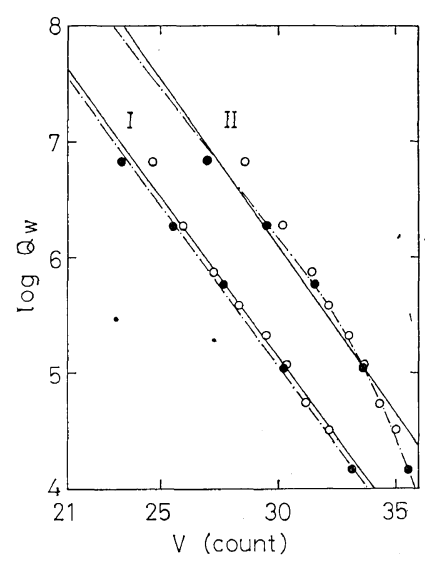

Figure 1. GPC calibration curves for the "universal" molecular size parameter, $Q_{w}=[\eta] M_{w}$ : closed circles, linear polystyrenes of L-series; open circles, fractionated samples of branched polystyrene, B-2 to B-9; solid line for column system I, eq 3; chain line for I, eq 4; solid line for II, eq 5; curved chain line for II, a most plausible calibration curve. 


$$
\begin{aligned}
& \log Q_{w}=13.47-0.278 \mathrm{~V} \\
& \log Q_{w}=13.40-0.278 \mathrm{~V}
\end{aligned}
$$

as indicated by the solid and chain lines, respectively. The separation between these two lines corresponds to a $15-\%$ difference in $M_{w}$, and it is rather difficult to discriminate between their accuracies by this figure alone. In system II, the calibration curve may not be linear as indicated by the chain curve. The resolution in the low-molecular-weight range is clearly spoiled by the removal of a $10^{4}$-column from this system. However, in the analysis of the present branched polystyrene samples, $\mathrm{C}$ to $\mathrm{K}$, the contribution of low-molecular-weight components to the total intrinsic viscosity is of rather minor importance, and accordingly, we may assume a linear relationship between $V$ and $Q_{w}$. This is indicated by the solid line in Figure 1, which is expressed as

$$
\log Q_{w}=14.78-0.289 \mathrm{~V}
$$

Similar calibration curves as above may be set between $V$ and $Q_{n}\left(=[\eta] M_{n}\right)$. However, they can not be recommended for use, for the peak count essentially correspondeds to $M_{w}$ rather than $M_{n}{ }^{7}$

\section{COMPUTER PROGRAM}

\section{Basic Equations}

We adopted the following set of equations as the basic equations for estimating the branching parameter $\lambda$ from intrinsic viscocity $[\eta]$ and GPC elution curve $f(V)$ :

$$
\begin{gathered}
\log Q_{i}=C-D V_{i} \\
Q_{i}=[\eta]_{i} M_{i}=K M_{i}{ }^{1+A} G\left(\lambda, M_{i}\right) \\
{[\eta]=\Sigma_{i} f_{i}[\eta]_{i}=K \Sigma_{i} f_{i} M_{i}^{A} G\left(\lambda, M_{i}\right)}
\end{gathered}
$$

Here $f_{i}$ represents the weight fraction of molecules which is eluted at the elution count $V_{i}$ and has the molecular weight $M_{i}$. Actually, $f_{i}$ was read on the elution curve at 0.5 intervals in count number. $C, D, K$, and $A$ are constants. Because of the mutual convertibility of $\lambda_{3}$ and $\lambda_{4}$ shown by eq 24 in Part I, we employ eq 2 for $G\left(\lambda, M_{i}\right)$ throughout this study.

\section{Computer Program}

Let us start with the simplest case, a linear polymer with $\lambda=0$ and $G(\lambda, M)=1$. In this case, the GPC elution curve $f_{i}\left(V_{i}\right)$ can be directly converted into the molecular weight distribution $f_{i}\left(M_{i}\right)$ with the aid of eq 6 and 7 . The intrinsic viscosity is then calculated by eq 8. The result $[\eta]_{\lambda=0}$ should be in agreement with the observed value $[\eta]_{\text {obs }}$ as far as the four constants $K, A, C$ and $D$ are properly selected. In the present case of polystyrene, values of $K$ and $A$ have been determined in Part $\mathrm{I}$ as

$$
K=1.14 \times 10^{-4}, \quad A=0.72,
$$

Accordingly, only two constants $C$ and $D$ are left to be determined.

In the case of branched polymers, however, $[\eta]_{\lambda=0}$ will be generally overestimated. Thus, we present an iterative computer method, in which $\lambda$ is incremented until agreement is achieved between $[\eta]_{\text {cale }}$ and $[\eta]_{\text {obs }}$.

A flow chart of the proposed computer program is shown in Figure 2. For a given polymer sample, the GPC data $f_{i}\left(V_{i}\right)$ and the observed value of $[\eta]$, VISOBS, are first fed to a computer. Then, calculate $[\eta]$ accroding to eq 6 to 8 with the assumption that $\lambda=0$. If the result, VISCAL, is in agreement with VISOBS within an allowance, say \pm 0.001 , it is printed together with the value of $\lambda$ used (i.e., $\lambda=0$ in the first run, $I=1$ ), and also with calculated values of various average molecular weights. Otherwise, the program proceeded to the right-hand column in the figure. Then, if (VISCAL - VISOBS) $>0.001$, LAMDA $(\lambda)$ is incremented by an amount of DELLAM $(\Delta \lambda)$, and we return to the left-hand column to recalculate [ $\eta]$ by using new value of $\lambda$. The value of $\Delta \lambda$ may be set as $1 \times 10^{-5}$ at the beginning. On the other hand, if (VISCAL-VISOBS) $<0.001$ in the first run with $I=1$, we simply return to the left-hand column without going to negative values of $\lambda$, for the parameter $\lambda$ should be always positive by its definition. However, if the case occurs in the run with $I \geq 2$, the used increment $\Delta \lambda$ is decreased by a factor of 0.1 , for such an underestimate of VISCAL occurs only when the used $\Delta \lambda$ was too coarse compared with the criterion that |VISOBS-VISCAL| $<0.001$. In this way, the calculation of $[\eta]$ can be iterated until agreement is achieved between 
GPC-Computer Analysis of Branched Polymers

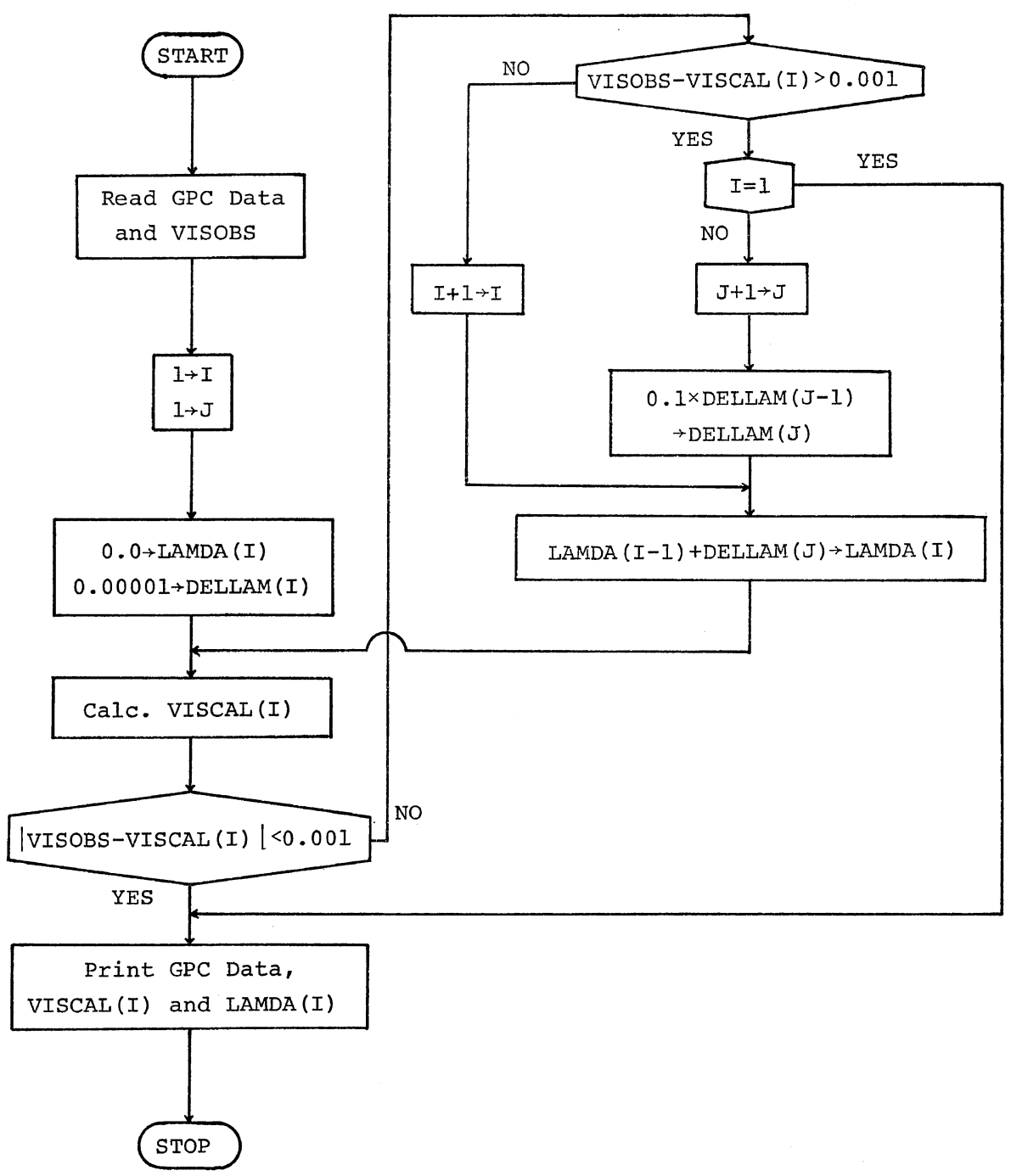

Figure 2. Flow chart of the proposed program for determination of the branching parameter $\lambda$.

VISCAL and VISOBS to within the allowance of $0.001 \mathrm{~d} l / g$.

The computer program used was written in Fortran IV for a FACOM 230-60. ${ }^{8}$ Running time was about one second per sample.

\section{RESULTS AND DISCUSSIONS}

\section{Linear Polystyrene}

To determine the most appropriate set of $C$ and $D$-values in eq 6 , and also to see how accurate is the predictions obtainable by this kind of GPC analysis, we treated the GPC data of two types of linear polystyrenes, L-series and MS-1, each having narrow- and wide-distributions of molecular weight, respectively. The results are summarized in Table IV. It will be seen that the first set of $C$ - and $D$-values corresponding to eq 3 leads to almost perfect predictions of $M_{w}$ and [ $\left.\eta\right]$, while the second set corresponding to eq 4 leads to underestimates, though to a small extent. On this basis, we selected eq 3 as the calibration line for the column system I.

With a careful selection of four constants, $A$, $K, C$, and $D$, as the present, the estimation 
Table IV. Analysis of the GPC elution curves for linear polystyrene samples obtained in the column system I

\begin{tabular}{|c|c|c|c|c|c|c|c|c|c|c|c|c|c|}
\hline \multirow[b]{2}{*}{ Sample code } & \multicolumn{3}{|c|}{ Observed } & \multicolumn{6}{|c|}{$C, 13.47 ; D, 0.278 ;$ eq 3} & \multicolumn{4}{|c|}{$C, 13.40 ; D, 0.278 ;$ eq 4} \\
\hline & $\begin{array}{c}M_{n} \\
\times 10^{-4}\end{array}$ & $\begin{array}{c}M_{w} \\
\times 10^{-4}\end{array}$ & $\begin{array}{l}{[\eta]} \\
\mathrm{d} l / g\end{array}$ & $\begin{array}{c}M_{n} \\
\times 10^{-4}\end{array}$ & $\begin{array}{c}M_{w} \\
\times 10^{-4}\end{array}$ & $\begin{array}{c}M_{z} \\
\times 10^{-4}\end{array}$ & $\frac{M_{w}}{M_{n}}$ & $\begin{array}{c}{[\eta]_{\lambda=0}} \\
\mathrm{~d} l / \mathrm{g}\end{array}$ & $\frac{[\eta]_{\lambda=0}}{[\eta]_{\mathrm{obs}}}$ & $\begin{array}{c}M_{n} \\
\times 10^{-4}\end{array}$ & $\begin{array}{c}M_{w} \\
\times 10^{-4}\end{array}$ & $\begin{array}{c}{[\eta]_{\lambda=0}} \\
\mathrm{~d} l / \mathrm{g}\end{array}$ & $\frac{[\eta]_{\lambda=0}}{[\eta]_{\mathrm{obs}}}$ \\
\hline L- 7 & 4 & - & 0.295 & & & & 1.11 & 30 & 1.03 & & .3 & 0.284 & 0.96 \\
\hline L- 1 & 15.7 & 16.5 & 0.668 & 14.0 & & 17.7 & 1.16 & 0.635 & 0.95 & 8 & 4.7 & 0.593 & 0.89 \\
\hline L- 3 & 40.1 & 44.4 & 1.3 & 32 & 42.2 & 48.0 & 1.29 & 1.26 & 0.95 & & 8.5 & 1.18 & 0.89 \\
\hline L- & 72.5 & 87.6 & 2.1 & 13.4 & 93.3 & 107 & & 2.23 & 1.05 & 68.7 & 85.1 & 2.09 & 0.98 \\
\hline L- & - & 189 & 3.62 & 98.1 & 193 & 254 & 1.97 & 3.69 & 1.02 & 89.5 & 173 & 3.44 & 0.95 \\
\hline MS-1 & - & $27 \pm 3$ & 0.843 & 10.1 & 26.4 & 49.4 & 2.61 & 0.846 & 1.00 & 9.2 & 24.1 & 0.790 & 0.94 \\
\hline
\end{tabular}

Table V. Analysis of the GPC elution curves for linear polystyrene samples obtained in the column system II

\begin{tabular}{ccccccccc}
\hline & \multicolumn{2}{c}{ Observed } & & \multicolumn{5}{c}{$C, 14.78 ; D, 0.289 ;$ eq 5} \\
\cline { 2 - 3 } \cline { 6 - 8 } Sample code & $\begin{array}{c}M_{w} \\
\times 10^{-4}\end{array}$ & $\begin{array}{c}{[\eta],} \\
\mathrm{d} l / \mathrm{g}\end{array}$ & & $\begin{array}{c}M_{n} \\
\times 10^{-4}\end{array}$ & $\begin{array}{c}M_{w} \\
\times 10^{-4}\end{array}$ & $\begin{array}{c}M_{z} \\
\times 10^{-4}\end{array}$ & $\begin{array}{c}{[\eta]_{\lambda=0}} \\
\mathrm{~d} l / \mathrm{g}\end{array}$ & $\frac{[\eta]_{\lambda=0}}{[\eta]_{\mathrm{obs}}}$ \\
\hline MS-1 & $27 \pm 3$ & 0.843 & & 13.9 & 27.1 & 51.2 & 0.868 & 1.05 \\
YML-1 & - & 0.767 & & 14.1 & 22.1 & 32.6 & 0.769 & 1.00 \\
YML-1 & - & 0.443 & & 9.0 & 11.8 & 15.4 & 0.499 & 1.13 \\
YML-7 & - & 0.990 & & 18.1 & 30.3 & 49.0 & 0.958 & 0.97 \\
\hline
\end{tabular}

of $[\eta]$ seems to be accomplished with an accuracy of $5 \%$ as is indicated by the values of $[\eta]_{\lambda=0} /[\eta]_{\text {obs }}$ in the table. At the same time, this seems to indicate the limit of accuracy achieved by the proposed method. Thus, we may conclude that in application to branched polymers, the estimation of $\lambda$ should not be pursued beyond this limit.

The values of $M_{w} / M_{n}$ obtained for the samples of L-series are slightly larger than the values given in Table III in Part I. The difference may be attributed to the so-called broadening effect in GPC. ${ }^{9}$ This effect becomes less significant for the sample MS-1 with a broad distribution of molecular weight, but still is not negligible.

The calibration line (eq 5) for the column system II was also tested with the linear samples, SM-1 and YML-series. The results are shown in Table V. Agreement between the calculated and observed values of $[\eta]$, and also of $M_{w}$, was satisfactory in all these samples but YML-4. The failure in the last sample is attributable to the poor approximation of eq 5 in the range of $10^{4}$-molecular-weights as observed in Figure 1, and it would be saved by the use of the chain line relationship instead of eq 5 . However, we did not try this modification.

\section{Branched Polystyrene}

Fractionated Samples and Their Blends. The proposed computer method was first applied to the fractionated samples of branched polystyrene, B-2 to B-9. The number- and weight-average molecular weights of these samples and the intrinsic viscosities in toluene at $30^{\circ} \mathrm{C}$ have already been determined in Part $\mathrm{I}$ as reproduced in Table VI. The branching parameter $\lambda$ has also been evaluated as $(0.8 \pm 0.2) \times 10^{-5}$. The molecular weight distributions were fairly sharp in these samples. This will be seen from the tabulated values of $M_{n}$ and $M_{w}$, and also from the GPC-elution curves illustrated in Figure 3. Although these cruves are of the blended samples explained below, the sharp peak appearing at $V=31 \sim 32$ can be assigned to B-3. The width of this peak was comparable with that for the linear polystyrenes with narrow-distribution, L-series.

The results of calculation are summarized in Table VI. Starting with $\lambda=0$, we obtain under- 


\section{GPC-Computer Analysis of Branched Polymers}

Table VI. Analysis of the GPC elution curves for fractionated samples of branched polystyrene B and their blends obtained in the column system I

\begin{tabular}{|c|c|c|c|c|c|c|c|c|c|c|c|c|}
\hline \multirow[b]{2}{*}{ Sample code } & \multicolumn{3}{|c|}{ Observed } & \multicolumn{3}{|c|}{ Calculated for $\lambda=0$} & \multicolumn{5}{|c|}{ Calculated with eq 3} & \multirow{2}{*}{$\begin{array}{c}\text { eq } 4 \\
\times \\
\times 10^{5}\end{array}$} \\
\hline & $\begin{array}{c}M_{n} \\
\times 10^{-4}\end{array}$ & $\begin{aligned} & M_{w} \\
& \times 10^{-4}\end{aligned}$ & $\begin{array}{l}{[\eta]} \\
\mathrm{d} l / g\end{array}$ & $\begin{array}{c}M_{w} \\
\times 10^{-4}\end{array}$ & $\begin{array}{c}{[\eta]_{\lambda=0}=} \\
\mathrm{d} l / \mathrm{g}\end{array}$ & $\frac{[\eta]_{\lambda=0}}{[\eta]_{\text {obs }}}$ & $\begin{array}{c}M_{n} \\
\times 10^{-4}\end{array}$ & $\begin{array}{c}M_{w} \\
\times 10^{-4}\end{array}$ & $\begin{array}{c}M_{z} \\
\times 10^{-4}\end{array}$ & $\begin{array}{c}\lambda \\
\times 10^{5}\end{array}$ & $\lambda M_{w}$ & \\
\hline B-2 & 7.4 & 9.0 & 0.360 & 8.1 & 0.385 & 1.07 & 7.5 & 8.7 & 9.7 & $(1.00)$ & $(0.9)$ & $(-)$ \\
\hline B-3 & 11.6 & 11.8 & 0.461 & 11.6 & 0.498 & 1.08 & 10.8 & 12.5 & 14.0 & $(0.82)$ & $(1.0)$ & $(0.13)$ \\
\hline B-4 & 18.8 & 18.9 & 0.627 & 16.5 & 0.641 & 1.02 & 14.2 & 16.9 & 19.1 & $(0.14)$ & $(0.2)$ & $(-)$ \\
\hline B-5 & 23.8 & 27.2 & 0.776 & 23.6 & 0.825 & 1.06 & 19.6 & 25.1 & 30.0 & $(0.30)$ & $(0.8)$ & $(-)$ \\
\hline B-6 & 35.4 & 39.9 & 0.948 & 33.8 & 1.066 & 1.12 & 27.0 & 38.2 & 47.4 & 0.46 & 1.8 & 0.18 \\
\hline B-7 & 53.5 & 64.2 & 1.15 & 50.5 & 1.41 & 1.23 & 37.3 & 62.7 & 89.2 & 0.69 & 4.3 & 0.40 \\
\hline B-8 & 78.0 & 125 & 1.50 & 78.4 & 1.94 & 1.29 & 66.6 & 103 & 142 & 0.68 & 7.0 & 0.45 \\
\hline B-9 & 156 & 307 & 2.16 & 168 & 3.30 & 1.53 & 117 & 265 & 460 & 0.80 & 21.0 & 0.60 \\
\hline $2+5$ & 11.3 & 18.1 & 0.568 & 15.8 & 0.602 & 1.06 & 9.8 & 16.9 & 26.1 & $(0.38)$ & 0.6 & $(-)$ \\
\hline $3+7$ & 19.1 & 38.0 & 0.810 & 31.5 & 0.960 & 1.18 & 16.4 & 38.4 & 80.4 & 0.70 & 2.7 & 0.36 \\
\hline $6+8$ & 48.8 & 82.5 & 1.220 & 66.9 & 1.68 & 1.38 & 37.4 & 95.6 & 193 & 1.04 & 9.9 & 0.73 \\
\hline $3+9$ & 21.2 & 157 & 1.290 & 86.6 & 1.84 & 1.43 & 18.4 & 133 & 432 & 0.75 & 10.0 & 0.53 \\
\hline
\end{tabular}

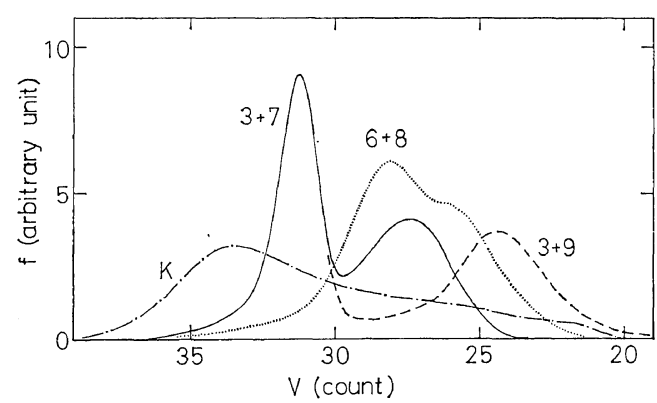

Figure 3. GPC elution curves of three binary blends of polystyrene fractions, B-3, 6, 7, 8, and 9 , and of unfractionated sample $\mathrm{K}$. The former is obtained in the column system $\mathrm{I}$, while the latter in II.

estimated values of $M_{w}$ and overestimated values of $[\eta]$. The ratio $[\eta]_{\lambda=0} /[\eta]_{\text {obs }}$ exceeds the $5-\%$ limit set in the last section, especially in the cases of four samples B-6 to B-9, and accordingly the calculation of $\lambda$ proceeded following the proposed computer program. The values of $\lambda$ thus determined nicely fitted the value, $(0.8 \pm 0.2) \times 10^{-5}$, already established in Part I. It is also notable that a rough agreement is regained between the calculated and observed values of $M_{w}$. On the contrary, $M_{n}$ remains underestimated. This may be attributed to the broadening effect in GPC. The values of $\lambda$ obtained for the samples B-2 to B-5 are of course less reliable, and therefore they are put in parentheses. However, it will be interesting to note that the ratio $[\eta]_{\lambda=0} /[\eta]_{\text {obs }}$ exceeded unity for all these samples. This is in contrast with the results obtained for the linear samples that the same ratio scattered rather randomly around unity as shown in Table IV, and indicates that these samples B-2 to B-5 also include branch points though to a small degree.

Now, before proceeding to the analysis of unfractionated samples, it seems desirable to test the accuracy of the method with a blended sample, since the GPC elution curve of the blend may not necessarily be a simple sum of the curves of component polymers.

Thus, we prepared four binary mixtures of B-fractions and performed GPC measurements on them. The mixing ratio was $50: 50$ for samples $2+5,3+7$ and $6+8$, and $50.9: 49.1$ for $3+9$, respectively, where the abbreviation $2+5$, for example, represents the mixture of B-2 and B-5. The GPC elution curves obtained for three samples, $3+7,6+8$ and $3+9$ are illustrated in Figure 3 as examples, two of them having typical bimodal shapes. The results of analysis of these curves are summarized in Table VI. In these cases, the values of $M_{n}$, $M_{w}$ and $[\eta]$ can be readily calculated from the values for component polymers as given in the first three columns in the table. Again for three samples $3+7,6+8$ and $3+9$, we obtain the values of $\lambda$ which are in agreement with 
Table VII. Analysis of the GPC elution curves for unfractionated samples of branched polystyrene, $\mathrm{C}$ to $\mathrm{K}$, obtained in the column system II

\begin{tabular}{ccccccccc}
\hline Sample code & $\begin{array}{c}M_{w, \lambda=0} \times 10^{-5} \\
\times 1 \eta]_{\lambda=0}\end{array}$ & $\begin{array}{c}M_{n} \\
\times 1]_{\mathrm{obs}}\end{array}$ & $\begin{array}{c}M_{w} \\
\times 10^{-5}\end{array}$ & $\begin{array}{c}M_{z} \\
\times 10^{-5}\end{array}$ & $\begin{array}{c}\lambda_{\mathrm{GPC}} \\
\times 10^{5}\end{array}$ & $\begin{array}{c}\lambda_{\text {anal }} \\
\times 10^{5}\end{array}$ & $\begin{array}{c}\lambda_{\mathrm{GPC}}^{\prime} \\
\times 10^{5}\end{array}$ \\
\hline C & 2.57 & 1.27 & 1.31 & 3.46 & 12.9 & 1.39 & 1.23 & 1.21 \\
$\mathrm{D}$ & 3.94 & 1.34 & 1.62 & 5.81 & 27.7 & 1.30 & 0.98 & 1.18 \\
$\mathrm{E}$ & 8.36 & 1.34 & 2.52 & 12.3 & 66.1 & 0.53 & 0.54 & 0.50 \\
F & 17.3 & 1.37 & 4.35 & 25.9 & 111 & 0.31 & 0.30 & 0.30 \\
G & 2.92 & 1.05 & 1.59 & 3.10 & 6.70 & $(0.14)$ & 0.29 & $(0.14)$ \\
H & 3.41 & 1.08 & 1.70 & 3.75 & 9.35 & $(0.20)$ & 0.42 & $(0.15)$ \\
I & 6.51 & 1.33 & 2.03 & 9.58 & 56.4 & 0.65 & 0.74 & 0.60 \\
J & 10.8 & 1.54 & 2.18 & 19.3 & 144 & 0.90 & 0.91 & 0.90 \\
K & 18.3 & 1.83 & 2.55 & 39.4 & 276 & 1.29 & 1.22 & 1.28 \\
\hline
\end{tabular}

the previous estimates, $(0.8 \pm 0.2) \times 10^{-5}$.

Finally, we repeated the same analysis as above, but with use of the calibration equation (eq 4) instead of eq 3 . Only the $\lambda$ values obtained are listed in the last column of Table VI. Although the data are not given in the table, $[\eta]_{\lambda=0}$ are decreased by a factor of about $6 \%$ from the tabulated values obtained with eq 3 and $M_{w, \lambda=0}$ by a factor of about $9 \%$, and the total $15-\%$ decrease corresponds to the difference 0.07 between two $C$ values in eq 3 and 4 . This effect gives rise to a decrease in $\lambda$, and to the negative values of $\lambda$ in the cases of samples B-2, B-4 and B-5. However, the rough constancy of $\lambda$ is retained for the samples, B-7, B- $8, \mathrm{~B}-9,3+7,6+8$, and $3+9$, which indicates the uniform density of branch points in these samples. The final values of $M_{w}$ are, of course, decreased by a factor of about $15 \%$ from those obtained with eq 3.

Unfractionated Samples. We are now in the position to test the method with unfractionated samples of branched polymers. For the purpose, we prepared nine polystyrene samples, $\mathrm{C}$ to $\mathrm{K}$, as shown in Table I. The GPC elution curves are extremely broad as illustrated by the curve $\mathrm{K}$ in Figure 3 .

The results obtained are shown in Table VII. The values of $[\eta]_{\lambda=0} /[\eta]_{\text {obs }}$ are fairly large when compared with those for the fractionated samples. Thus, the reasonably reliable estimation of $\lambda$ is obtainable for all samples but $\mathrm{G}$ and $\mathrm{H}$. The obtained values of $\lambda$ show close agreement with the values $\lambda_{\text {anal }}$ which are evaluated from consideration of polymerization kinetics and

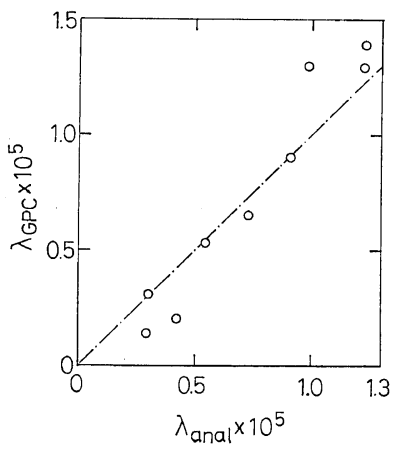

Figure 4. Comparison of two estimates of the branching parameter $\lambda$ obtained for unfractionated samples, $\mathrm{C}$ to $\mathrm{K}$.

already given in Table I. This is illustrated in Figure 4, where the plots of $\lambda_{\mathrm{GPC}}$ against $\lambda_{\text {anal }}$ fall in the proximity of the diagonal. As already mentioned, these unfractionated samples are not raw samples, but the ones treated by filtration through a GPC filter. As a result, a microgel component has been removed from each sample. Nevertheless, we obtained the agreement between $\lambda_{G P C}$ and $\lambda_{\text {anal }}$. This fact indicates that the removed component must be characterized by the same density of branch points as the remaining main component, and that removal or addition of a component having the same branching density does not affect the result of the present GPC analysis as was the case for the blend samples of B-series.

The above calculation was based on the viscosity constants given in eq 9 . Now, if these constants are modified as

$$
K=9.77 \times 10^{-5}, \quad A=0.73
$$




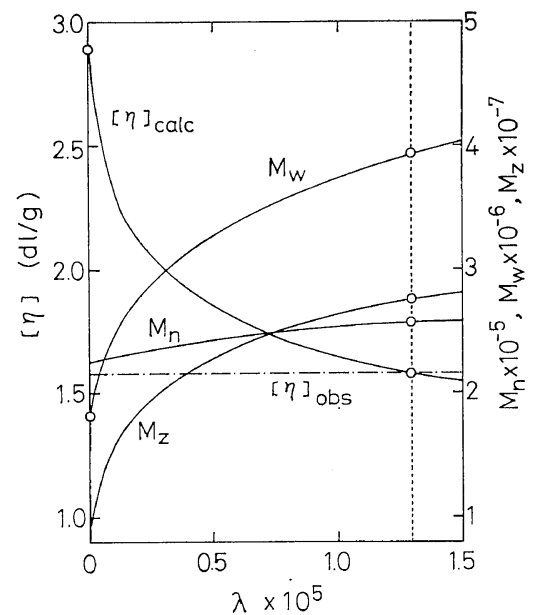

Figure 5. Variation of the calculated values of [ $\eta], M_{n}, M_{w}$, and $M_{z}$ during the course of the iterative computation: open circles, initial values of $[\eta]$ and $M_{w}$ corresponding to $\lambda=0$, and final values of $[\eta], M_{n}, M_{w}$, and $M_{z}$ corresponding to $\lambda=1.29 \times 10^{-5}$; chain line, the level of $[\eta]_{\text {obs }}$.

we obtain the values listed as $\lambda_{G \mathrm{PC}}^{\prime}$ in Table VII, which are practically indistinguishable from $\lambda_{\mathrm{GPC}}$. The variation of the Mark-HouwinkSakurada constants of this order does not contribute any significant effect on the estimation of $\lambda$.

In Figure 5, we illustrate as an example the variations of four quantities, $[\eta], M_{n}, M_{w}$, and $M_{z}$, obtained in the course of the program computation on the sample $\mathrm{K}$. The curve for $[\eta]_{\text {calc }}$ intersects with the chain line representing $[\eta]_{\text {obs }}$ at $\lambda=1.29 \times 10^{-5}$. Correspondingly, we obtain the values indicated by circles for three average molecular weights. It will be seen in the figure that a $3-\%$ variation in $[\eta]_{\mathrm{obs}}$ gives rise to a $15-\%$ variation in $\lambda$ and 3-\% variations in $M_{w}$ and $M_{z}$, respectively. On the other hand, $M_{n}$ is rather insensitive to these variations.

According to the theory of branching, ${ }^{10,11}$ the weight-average number of branched points per molecule $m_{w}$ and various ratios of molecular weights can be expressed in terms of a quantity $\gamma$ often called the branching parameter;

$$
\begin{gathered}
m_{w}=\lambda M_{w}=\gamma /(1-\gamma) \\
M_{w} / M_{n}=2(1-\gamma / 4) /(1-\gamma) \\
M_{z} / M_{w}=3 / 2(1-\gamma) .
\end{gathered}
$$

As is well known, $\gamma$ is zero for linear polymers and attains unity at the incipient gelation point. In this meaning, $\gamma$ represents the extent of branching reaction. For highly branched polymers with $r \simeq 1$, we obtain

$$
M_{w} / M_{n}=M_{z} / M_{w}=3 / 2 \delta, \quad \delta=1-\gamma
$$

which is the behavior characteristic of the so-called log-normal distribution.

It has also been shown by Kilb ${ }^{11}$ that the viscosity ratio $[\eta]_{b} /[\eta]_{1}$ for an unfractionated sample of randomly branched polymer is expressed by a universal function of $\gamma$. Here $[\eta]_{\mathrm{b}}$ represents the intrinsic viscosity of the branched sample and $[\eta]_{1}$ represents that of a linear sample with the same molecular weight $M_{w}$ as the branched sample and with the most probable distribution of molecular weight. This function is indicated by the curve in Figure 6. An experimental proof of this relationship has recently been given by Kamada and Sato, ${ }^{12}$ and it can be utilized to estimate $\lambda$ from measurements of $[\eta]_{b}$ and $M_{w}$. However, for a highly branched sample with large $\gamma$, light-scattering determination of $M_{w}$ becomes intolerably difficult due to abnormally large scattering from huge particles, and this method for estimatson of $\lambda$ becomes impractical.

Now, returning to our samples, we can readily evaluate $\gamma$ by substituting the obtained values of $M_{w}$ and $\lambda$ into eq 11 . This is done as shown in Table VIII. Here we used $\lambda_{\text {anal }}$ rather than $\lambda_{\mathrm{GPC}}$ in the calculation, for $\lambda_{\mathrm{GPC}}$ is less reliable for some samples such as $\mathbf{G}$ and $\mathbf{H}$ than is $\lambda_{\text {anal }}$. The values of $[\eta]_{1}$ are obtainable

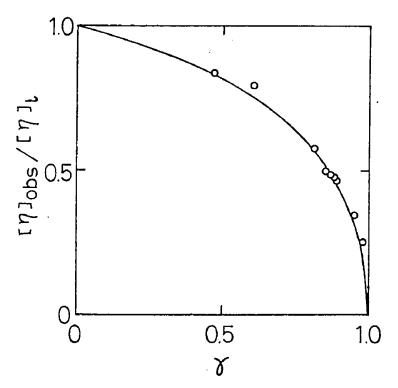

Figure 6. Viscosity ratio $[\eta]_{\mathrm{b}} /[\eta]_{1}$ of polydisperse randomly branched polymers as a function of the extent of branching reaction $\gamma$ : open circles, calculated values for unfractionated samples, C to $\mathrm{K}$. 
Table VIII. Molecular-weight and branch-point distributions in unfractionated polystyrenes, $\mathrm{C}$ to $\mathrm{K}$

\begin{tabular}{|c|c|c|c|c|c|c|c|c|}
\hline \multirow{2}{*}{ Sample code } & \multirow{2}{*}{$\begin{array}{c}{[\eta]_{\mathrm{obs}},} \\
\mathrm{d} l / \mathrm{g}\end{array}$} & \multirow{2}{*}{$\lambda_{a} M_{w}$} & \multirow{2}{*}{$\gamma$} & \multirow{2}{*}{$\begin{array}{l}{[\eta]_{1}} \\
\mathrm{~d} l / \mathrm{g}\end{array}$} & \multirow{2}{*}{$\frac{[\eta]_{\mathrm{b}}{ }^{a}}{[\eta]_{1}}$} & \multicolumn{2}{|c|}{$M_{w} / M_{n}$} & \multirow{2}{*}{$\begin{array}{c}M_{z} / M_{w}, \\
\text { GPC }\end{array}$} \\
\hline & & & & & & GPC & eq 12 & \\
\hline $\mathrm{C}$ & 0.639 & 4.3 & 0.81 & 1.11 & 0.58 & 2.6 & 9.3 & 3.7 \\
\hline $\mathrm{D}$ & 0.797 & 5.7 & 0.85 & 1.61 & 0.50 & 3.6 & 13.0 & 4.8 \\
\hline E & 1.340 & 6.6 & 0.87 & 2.76 & 0.49 & 4.9 & 12.0 & 5.4 \\
\hline F & 2.220 & 7.8 & 0.89 & 4.73 & 0.47 & 6.0 & 14.1 & 4.3 \\
\hline G & 0.858 & 0.9 & 0.47 & 1.02 & 0.84 & 2.0 & 2.6 & 2.2 \\
\hline $\mathrm{H}$ & 0.935 & 1.6 & 0.61 & 1.17 & 0.80 & 2.2 & 3.1 & 2.5 \\
\hline I & 1.125 & 7.1 & 0.88 & 2.31 & 0.49 & 4.7 & 11.2 & 5.9 \\
\hline $\mathrm{J}$ & 1.315 & 17.6 & 0.95 & 3.82 & 0.34 & 8.9 & 30.5 & 7.4 \\
\hline $\mathrm{K}$ & 1.58 & 48.1 & 0.98 & 6.39 & 0.25 & 15.4 & 75.5 & 7.0 \\
\hline
\end{tabular}

a $[\eta]_{\mathrm{b}}$ is identical to $[\eta]_{\mathrm{obs}}$ in the first column.

from $M_{w}$ with the aid of the [ $\left.\eta\right]$ vs. $M_{w}$ relationship, eq 22 in Part I. Then, the ratio $[\eta]_{\mathrm{b}} /[\eta]_{1}$ can be plotted against $\gamma$ as shown by the open circles in Figure 6. It is notable that even for filtered samples as in the present case, the obtained values of $[\eta]_{\mathrm{b}} /[\eta]_{1}$ retain agreement with the theoretical curve due to Kilb. The values of $M_{w} / M_{n}$ calculated by eq 12 , however, deviated appreciably from the values directly estimated from GPC. This is of course due to the effect of filtration, though quantitative analysis of the effect can not be made because of the lack of informations on $M$ of untreated samples. Finally, we note that approximate equality between two ratios, $M_{w} / M_{n}$ and $M_{z} / M_{w}$, is maintained in all the filtered samples but $\mathrm{K}$.

In conclusion, the proposed computer program seems to. offer a simple and quick method for estimating the degree of branching $\lambda$ and the weight-average molecular weight $M_{w}$ with a reasonable accuracy, i.e., about $15 \%$ for $\lambda$ and $10 \%$ for $M_{w}$. A rough estimate is also obtainable simultaneously for the number- and $z$-average molecular weights $M_{n}$ and $M_{z}$.

Acknowledgement. We wish to thank $\mathrm{Mr}$. Shozo Nishida of the Japan Synthetic Rubber Company, Ltd., for his assistance in GPC analysis.

\section{REFERENCES}

1. Z. Grubisic, P. Rempp, and H. Benoit, $J$. Polym. Sci., Part B, 5, 753 (1967).

2. I. Wild and R. Guliana, J. Polym. Sci., Part A-2, 5, 1087 (1967).

3. E. F. Cassasa and Y. Tagami, Macromolecules, 2, 526 (1969).

4. E. E. Drott and R. A. Mendelson, paper presented at 6th International GPC Seminar, Miami Beach, 1968; J. Polym. Sci., Part A-2, 8, 1361, 1373 (1970).

5. Reports from the Committee, Progr. Polymer Phys. Japan, 4, 141 (1961); 14, appendix-1 (1971).

6. Y. Toyoshima and H. Fujita, J. Phys. Chem., 68, 1378 (1964).

7. G. Meyerhoff, J. Polym. Sci., Part C, 21, 31 (1968).

8. M. Kurata, J. Soc. Rubber Ind., Japan, 45, 105 (1972), in Japanese.

9. M. Iwama, N. Tagata, and T. Homma, Kogyo Kagaku Zasshi (J. Chem. Soc. Japan, Chem. Ind. Sect.), 72, 926 (1969), in Japanese.

10. W. H. Stockmayer, J. Chem. Phys., 11, 45 (1943); 12, 125 (1944).

11. R. W. Kilb, J. Polm. Sci., 38, 403 (1959).

12. K. Kamada and H. Sato, Polymer J., 2, 489 (1971). 